\title{
LA DECORAZIONE SCULTOREA DELLE VILLAE ROMANE IN BAETICA
}

\author{
THE SCULPTURAL DECORATION OF ROMAN VILLAE IN BAETICA
}

\author{
MARIA CRISTINA NAPOLITANO*
}

Resumen: En los últimos treinta años ha habido un aumento del interés sobre las villae romanas de Hispania. Esta situación ha producido un desarrollo del análisis teórico, así como el aumento de excavaciones ha permitido nuevos descubrimientos arqueológicos. Un análisis de las esculturas encontradas en estas villae permite la posibilidad de obtener consideraciones sobre la cultura figurativa, los gustos y las tendencias de la sociedad de la Baetica en época imperial. Además, gracias a este análisis se obtiene una nueva visión sobre la distribución cuantitativa de los tipos, basados en el cálculo numérico de las esculturas encontradas.

Palabras clave: Arqueología. Baetica. Escultura romana. Distribución cuantitativa

Tra i documenti più indicativi della straordinaria ascesa della Spagna in epoca imperiale vanno annoverate alcune villae scavate nelle città della provincia Baetica (Gorges 1979; Fernández-Castro 1982), seconda per numero di tali stabilimenti soltanto alla Tarraconense. La Baetica possiede il 23\% del totale delle villae della penisola Iberica, disposte con regolarità tra il Conventus Hispalensis e il Conventus Astigitanus, in cui sono la maggior parte degli stanziamenti (88\%). Più di centoquaranta stabilimenti agricoli sono stati censiti fino ad ora, nonostante sembri poco rispetto

\footnotetext{
* Piazza Spartaco, 7. 80053 Castellammare di Stabia (NA). Italia. Correo-e: napolitano.cristina@libero.it
}

\begin{abstract}
The last thirty years there has been an increase of interest in the Roman villae in Hispania. This situation has produced a development of surface analysis and excavations, which have prompted some new archaeological discoveries. An analysis of the overall sculptures found allows some considerations on the figurative culture, the tastes and trends of the Baetica, during the imperial age. This analysis allows a quantitative distribution of types, based on the numerical computation of the sculptures found.
\end{abstract}

Key words: Archaeology. Baetica. Roman Sculpture. Quantitative distribution.

all'attività che questa zona pare effettivamente aver conosciuto.

Nella Valle del Guadalquivir, in epoca tardo-repubblicana, la conquista romana accelerò il fenomeno di diffusione delle villae, la cui presenza nella zona è da segnalare già a partire dal I sec. a.C. (Balil 1959-1960: 346-359; Gorges 1979: 26-28). Tale sistema, inizialmente con una forma urbanistica elementare, si sviluppò in determinate zone della regione catalana, a Carthago Nova e nella valle del Guadalquivir, in cui varie erano le attrattive per gli emigranti italici.

Nonostante tale abbondanza, al momento di studiare il fenomeno delle villae in Baetica l'archeologia si confronta, generalmente, con la scarsezza e il cattivo stato di conservazione dei resti materiali esistenti, 
distrutti per l'uso continuato nell'antichità del sito stesso o perché sofferenti degli attacchi dell'erosione naturale e della spoliazione. Tale situazione non ha comunque impedito, nell'ultimo trentennio, lo sviluppo di un certo interesse in Spagna, per questo tipo di stabilimento romano. Come conseguenza si è registrato un aumento delle analisi di superficie e scavi - anche in Andalusia - da cui sono derivate nuove scoperte archeologiche (Carrillo 1993: 233-257; Rodríguez Oliva 1993: 23-61; Baena 1994: 42-43; Cerrillo 1995: 17-26).

L'analisi complessiva delle sculture rinvenute consente la formulazione di riflessioni e di considerazioni sulla cultura figurativa, sui gusti e sulle tendenze della società betica di età imperiale.

Nel grafico I si riassumono i dati quantitativi, desunti dall'analisi delle fonti archeologiche - pubblicazioni e inventari dei siti - relativi alle villae romane da cui provengono i pezzi scultorei presi in esame (per i dati statistici relativi alla presenza di villae, individuate e/o indagate mediante scavo o prospezioni di superficie, nelle provincie di Cádiz, Almería, Granada e Málaga: Fornell 2005).

In ordine geografico-provinciale da est ad ovest, in funzione dell'allontanamento progressivo dalla valle del Guadalquivir, la prima provincia della Baetica è Córdoba (Ponsich 1987): in tale provincia soltanto la villa di El Ruedo (Almedinilla) è stata scavata e i risultati integralmente pubblicati (Vaquerizo 1990: 125-154; Koppel 1995: 34; Vaquerizo e Carrillo 1995: 121-155; Vaquerizo e Noguera 1997). Tale villa possiede il corpus di pezzi scultorei più cospicuo (29 frammenti analizzati).

La provincia con il maggior numero di villae è Málaga: 15 villae hanno restituito frammenti scultorei; tra queste, la villa de la Estación ha il maggior numero di pezzi (11 in totale). Di seguito vi è Granada, in cui sono situate le villae di Lecrín (Beltrán e Loza 2001: 538; 2003: 95-97) e Paulenca (Santero 1975: 227-268), da cui provengono alcuni dei frammenti scultorei esaminati. Segue Almería, con criterio decrescente nel numero delle villae testimoniate. In questa provincia è collocata una delle villae in analisi: El Villar (Chirivel) (Rodríguez Oliva 1993: 40; Koppel 1995: 36.). La provincia con minor numero di villae romane è Huelva (di cui in analisi è la villa "El Canto"). Infine Jaén - con il Cortijo de los Robles (Baena 2007: 245-248) - sulla cui appartenenza al territorio provinciale betico tuttora si discute (Corzo e Jiménez 1980: 21-47).

Delle villae sopraindicate sono riassunti, nella tavola sinottica 1, dati che riguardano, per ciascuna villa: luogo, sito, cronologia, tipologia, tipo di pianta e numero delle sculture rinvenute ed analizzate.

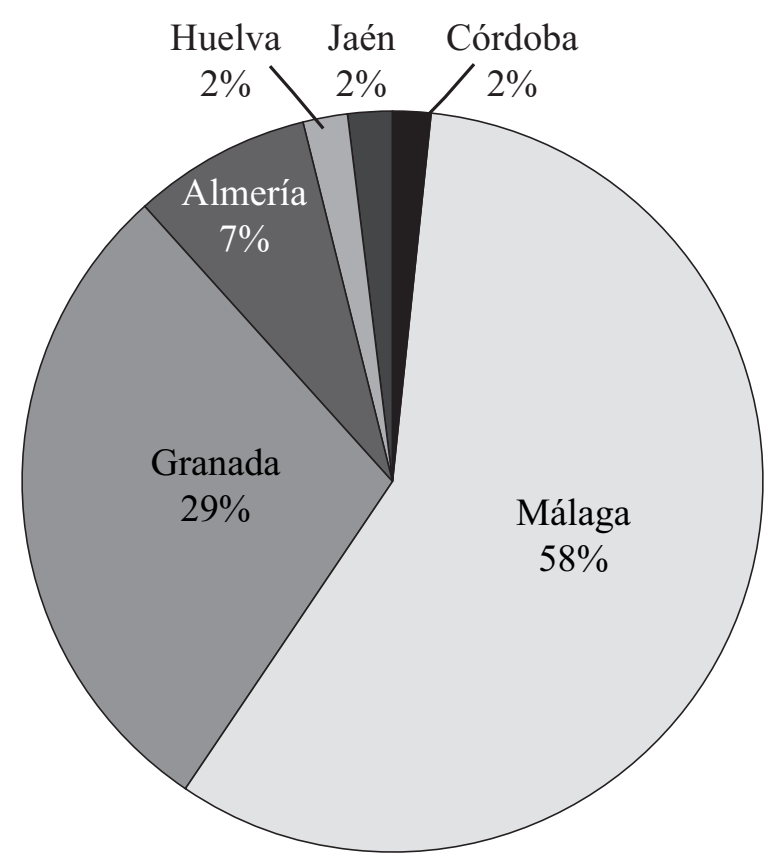

Grafico I. Distribuzione dei siti per provincia relativi a villae

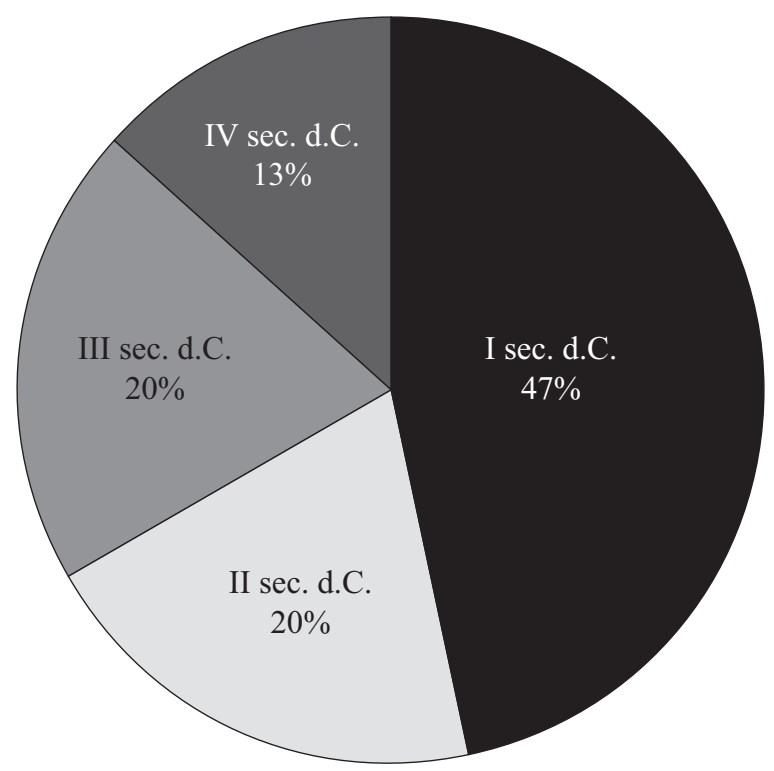

Grafico II. Cronologia delle villae

Dal punto di vista cronologico la maggiore diffusione di impianti relativi a villae in Baetica si verifica a partire dal I sec. d.C. (Grafico II), coincidendo con l'impero di Augusto e Vespasiano e in evidente relazione 

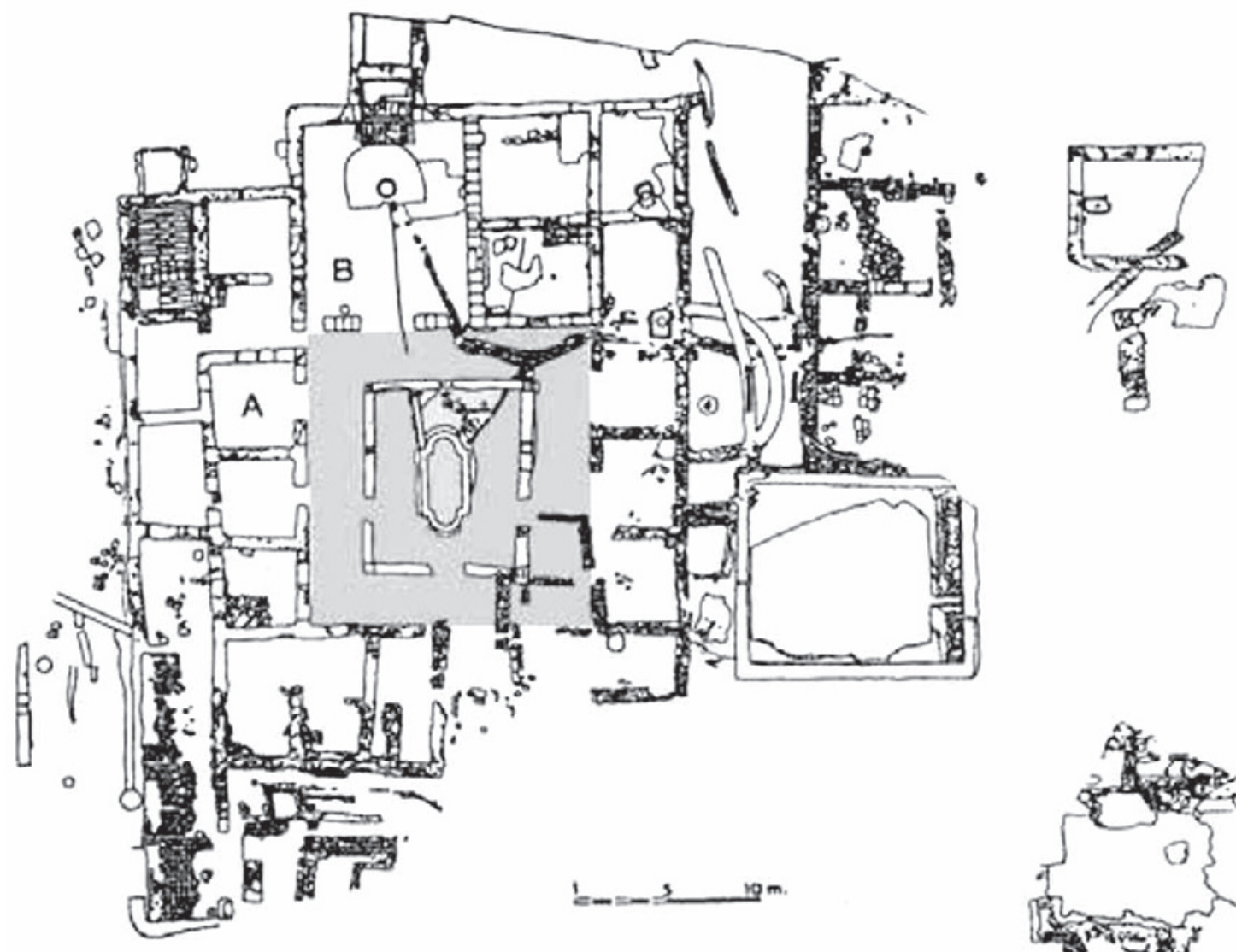

Figura 1.

con l'intensificarsi del processo di urbanizzazione in Hispania, in generale e nella Baetica in particolare.

Sulla base dello studio delle piante delle villae scavate o parzialmente scavate, si denota una presenza massiccia di dimore rustico/signorili. Tale definizione bivalente indica un distacco solo parziale - a differenza di quanto accadeva in Campania - della villa residenziale dall'attività agricola, da cui chiaramente il proprietario traeva ricchezza e sostentamento. Nella costa malagueña, sono note una serie di villae che sviluppano, insieme a quella agricola, attività derivate dalla pesca: Sabinillas (Manilva) (Posac e Rodríguez Oliva 1979: 129-145), Faro di Torrox (Rodríguez Oliva 1978b).

In ultimo sono da menzionare alcune villae ritenute di tipo rustico, in cui però la scarsezza dei resti architettonici conosciuti fino ad ora, non permette ulteriori classificazioni. Tra queste rientra la villa di Paulenca, in cui la parte scavata corrisponde soltanto alla pars fructuaria.
Le piante degli edifici nel territorio betico si conformano, in linea generale, al modello greco della casa a pastàs-peristilio. Alcuni esempi sicuri di villa a pianta distribuita intorno ad un peristilio sono a Córdoba: El Ruedo (fig. 1), Casa di Mitra (Cabra) (Blanco et al. 1972: 297-319); a Málaga: La Estación (Romero et al. 2006: 239-258; Rodríguez Oliva 2010: 63), Finca de El Secretario (Fuengirola) (Atencia e Sola 1978: 73-84), Torre de Benagalbón (Rincón de la Victoria) (Salado 2005: 353-378).

Uno dei pochi casi di villa a pianta composita irregolare è Faro de Torrox (Málaga). Tale villa risulta essere l'unica con questo tipo di pianta nel sud della Spagna, essendo questa una tipologia tipica delle villae del nord.

È forse attestata una villa "a corridoio" o Portikusvilla che prevede, rispetto alla semplice villa chiusa in un rettangolo, l'aggiunta di una galleria in facciata: si ritiene che El Villar presenti una pianta di questo tipo, 


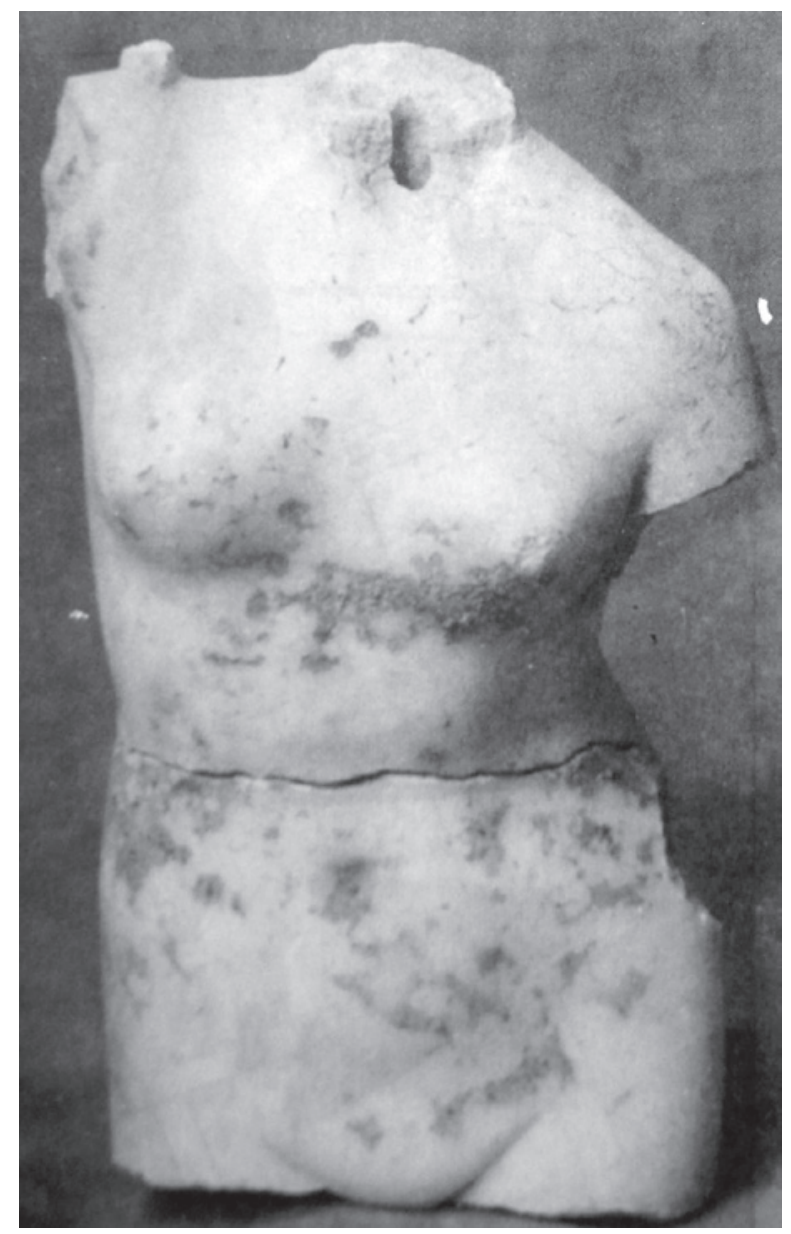

Figura 2. Statua di Afrodite

nella parte signorile dell'impianto - in attesa che si scavi totalmente e si analizzino le parti dipendenti del settore sud.

Sulla base di una ripartizione quantitativa dei tipi, basata sul computo numerico delle sculture rinvenute nelle villae romane della Baetica e di cui esistono pubblicazioni, si riscontrano, in vari formati e in diverse tipologie: statue di divinità, putti e animali, hermae, bronzi, gruppi, oscilla; in misura minore ritratti di personaggi pubblici o privati, pinakes e rilievi (Grafico III). Analizzando le percentuali nel dettaglio le statue e le hermae risultano particolarmente numerose.

Compaiono nelle villae betiche sculture dedicate a divinità spogliate del carattere sacro originale, ma che non hanno perso il favore della gente. Statue di divinità olimpiche (per lo più Afrodite ed Artemide, ma anche Apollo, Eros, Ermes) raramente a misura originale, allegorie e personificazioni sono tutte ispirate a modelli greci classici ed ellenistici (Fuengirola, Chirivel, Cabra, Almedinilla).

Tra queste si menziona una statua di Afrodite (fig. 2) (Mendoza et al. 1985: 897-902, fig. 3a; Beltrán e Loza 2001: 538, n. 15; 2003: 95-97, n. 2), proveniente dalla villa de Lecrín, il cui movimento degli arti permette di ipotizzare che si tratti di una Afrodite del tipo Anadyomene, rappresentata nell'atto di strizzarsi le chiome. Si vedano per confronto: la statuetta di Venere proveniente dalla Casa del Centenario a Pompei (AA.VV. 2008: 196-197, n. D49) e due esemplari provenienti dall'Egitto, oggi a Cambridge, copie da collocare fra il tardo Ellenismo e la prima età imperiale (Budde e Nicholls 1964: 53-54, n. 85-86, tav. 27).

Il busto in bronzo di Atena da Torrox (Rodríguez Oliva 1978b: 43, n. 3), oggi perduto, può invece rientrare nei temi comuni alle collezioni romane, intese come immagini emblematiche intorno alle quali si intrecciano le espressioni più elevate dell'intelletto umano; un possibile confronto può essere rappresentato dall'esemplare in marmo proveniente dalla villa di Cassio a Tivoli, oggi ai Musei Vaticani (Giuliani 1966: 197).

Degli orti saranno proprie le divinità protettrici del ciclo vegetale come Pan da Aguilar de la Frontera (Rodríguez Oliva 1993: 43) o il Priapo portatore di frutti (AA.VV. 1981-1997, V, s.v. kairoi tempora anni: 905, fig. 147) da Bobadilla (Baena 1980-81: 141 ss., lam. II) e da Alameda (Baena 1980-81: 141-150). Tali pezzi possono essere confrontabili con la statua di fauno con frutti nella nebride proveniente dal giardino della Casa del Poeta Tragico a Pompei (Jashemski 1993: 133, fig. 143; AA.VV. 2008: 84, B18) e con una statua definita di Silvano a Roma, ai magazzini dei Musei Vaticani (Kaschnitz-von Weinberg 1937: 97, tav. XXXV, fig. 199). Il Priapo proveniente da Alameda ha la particolarità di essere in Baetica l'unico nel suo genere.

Per alcune specifiche caratteristiche, quali la presenza di una figura di fanciullo nella parte inferiore destra - un probabile erote stagionale - il Priapo è inquadrabile nel "Gruppo G" di Hertel, in cui il dio appare circondato da quattro eroti che personificano le stagioni (Rodríguez Oliva 1987: 127). Somigliante al pezzo in questione, dal punto di vista stilistico, vi è solo un mosaico proveniente da Bobadilla - Antequera, che rappresenta un Priapo con frutta e spighe mantenute sulla veste (Portillo et al. 1985: 201, n. 22).

Figure puramente ideali, con scopo decorativo, sono invece il Dioniso adolescente da Aguilar de la Frontera (Rodríguez Oliva 1993: 43; Koppel 1995: 34; Vaquerizo 1995: 82-83), i frammenti di ali di eroti (Vaquerizo e Noguera 1997: 174, n. 17-18-19-20; Beltrán 


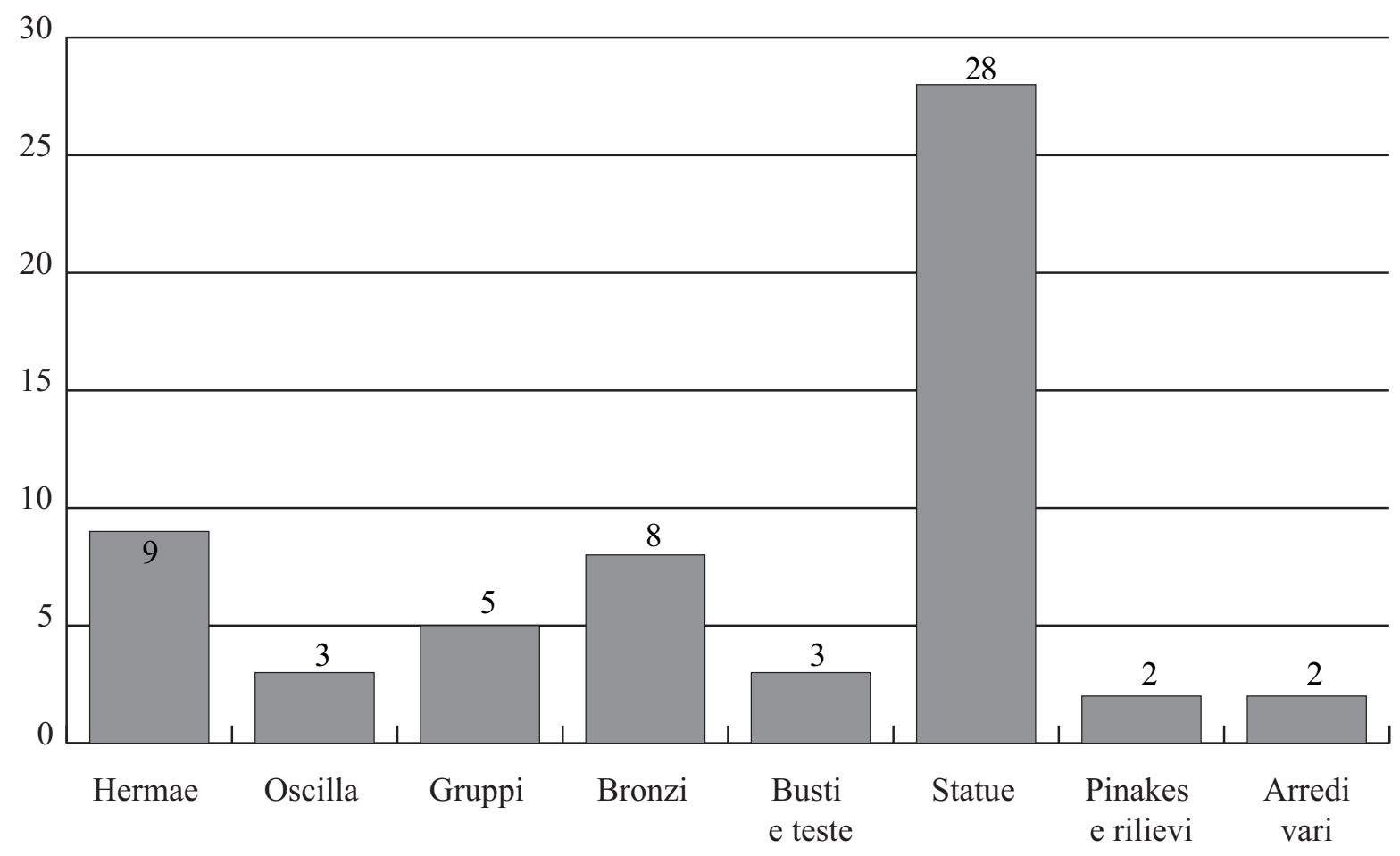

Grafico III. Distribuzione delle sculture per tipologia

e Loza 2003: 86, n. 14-15-16) e lo Hypnos da Almedinilla (AA.VV. 1990: 258 n. 179 bis; Vaquerizo e Noguera 1997: 150, n. 12): rappresentazioni di divinità infantili che rispondono al gusto ellenistico per le immagini di bimbi in diverse attitudini (Bieber 1961: 136138; Vorster 1983).

L'ambiente più adeguato per questi pezzi è l'abitazione privata (Neudecker 1988: 32), soprattutto giardini. Come sostiene P. Rodríguez Oliva (Rodríguez Oliva 1993: 43, n. 22) si tratta di pezzi «adatti alla decorazione domestica e lontani dalle rappresentazioni cultuali delle stesse divinità in età adulta»; «tipi ausiliari disegnati specificamente come fontane» invece sono per E. J. Dwyer (1982: 126): sembra chiaro che nessuna di queste statue dovette servire come immagine di culto domestico.

È appena accennata la presenza di divinità orientali nelle villae betiche, se non per l'unico caso del Mitra di Cabra (García y Bellido 1952: 389; Rodríguez Oliva 1993: 40, tav. 1; Vaquerizo 1995: 85, n. II.1).

Sono da menzionare nel novero della statuaria di piccolo formato delle raffigurazioni di animali, in marmo o in bronzo: la pantera da Casilla de la Lámpara a Montilla (Loza 1993a: 102; Vaquerizo 1995: 89-90, tav. 2) o il leone da Torrox (Rodríguez Oliva 1977: 21, fig. $24 ; 1978: 47$, tav. II) che rientrano a pieno nel gusto di riprodurre animali, molto vivo nelle città vesuviane. La presenza di esemplari del genere utilizzati come elementi da fontana è attestata a Pompei nella casa I, 329 da cui proviene una statuetta di cane per fontana (AA. VV. 2008: 32, n. A12).

Diverso è invece il caso del piccolo ippopotamo, databile nel II sec. d.C. (Romero et al. 2006: 254; Rodríguez Oliva 2010: 73), rinvenuto nel peristilio della villa de La Estación, probabilmente adeguato alla moda egittizzante romana che spesso si volse a questo tipo di animali acquatici evocanti paesaggi nilotici (si vedano due sculture di piccolo formato nella Ny Carlsberg Gliptothek di Copenhagen e nel deposito dei Garibaldini di Roma: De Nuccio e Ungaro 2002: 361-365).

L'altra tipologia scultorea che detiene la percentuale maggiore tra gli apparati decorativi delle villae romane della Baetica è quella delle hermae. Risulta necessario sottolineare che la maggior parte di tali pezzi proviene dalle villae di: Sabinillas, Villanueva de Algaidas (Corrales 2007: 206), Cortijo del Tajo (Corrales 2007: 207), Benagalbón (fig. 3), Bobadilla. Tali piccole sculture sono in Hispania, opera esclusiva della Baetica; fino ad oggi, quelle conosciute provengono per lo più da lì: Cártama (Málaga), Arcos de la Frontera 


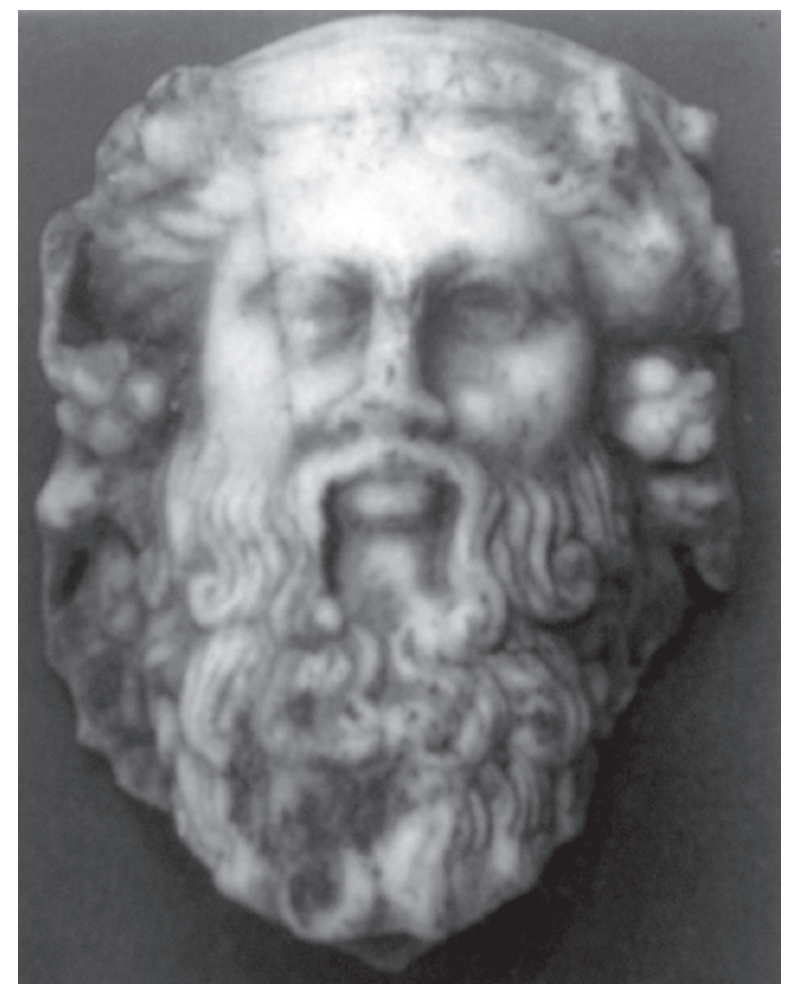

Figura 3. Herma de Benagalbón

(Cádiz), Tocón (Granada), Beas de Segura (Jaén), Espera (Cádiz), Montilla (Córdoba), Torrox (Málaga) (Portillo et al. 1985: 184-217) - e derivano dalle hermai greche. Sono solite rappresentare Dioniso, Hermes, Pan, Menadi, Arianna, Sileno, Priapo, fauni.

Facilitato nella diffusione da un basso costo ed una grande accessibilità, questo tipo scultoreo era facilmente collocabile sopra pilastrini posti nei peristili, giardini o altri spazi aperti delle villae.

Dal punto di vista quantitativo le sculture bronzee seguono le hermae. Esempi particolarmente significativi tra i pezzi in bronzo sono: lo Hypnos da El Ruedo (Vaquerizo e Noguera 1997: 150, n. 12; Baena 2004: 323); il Dioniso adolescente (Koppel 1995: 34; Vaquerizo 1995: 82-83) da las Minas (Rodríguez Oliva 1993: 44; Vaquerizo 1995: 82-83; Baena 2004: 323); l’Efebo da la Finca Las Piletas (Antequera) (Baena 2004: 323; AA.VV. 2011) il cui parallelo più vicino proviene da Volubilis (Michon 1933: 119), oggi a Rabat.

Le somiglianze tra $\mathrm{i}$ due pezzi, in particolare per la pettinatura avvolta da una corona di edera, lascia pensare che facciano capo entrambi al medesimo archetipo perduto e che in essi si ritrovino delle copie vicine all'originale greco di età periclea, dal quale discendono numerosi altri pezzi, come quello scoperto, contemporaneamente a quelli di Antequera e Volubilis, in Via dell'Abbondanza a Pompei (Picard 1939: 346, fig. 148).

Un pezzo di categoria comparabile all'Efebo di Antequera è la testa bacchica proveniente dal Cortijo de los Villares di Serrato, ispirata ad un prototipo prassitelico di secolo IV a.C.; è databile alla fine del I sec. d.C. o ai primi anni del II sec. d.C., per le sue caratteristiche e per le comparazioni con altri pezzi simili (AA.VV. 1990: 100 e 253, n. 172).

I pezzi che maggiormente si avvicinano alla testa di Serrato sono quelli del Museo Capitolino (AA.VV. 1981-1997, III, s.v. Dionysos: 444, n. 203; Stuart Jones 1968: 96, n. 17, tav. 27), Venezia (Pochmarski 1974: 163, fig. 40-B) e Corinto (AA.VV. 1981-1997, s.v. Dionysos: 444, n. 200; Pochmarski 1974: 159, fig. 38-A).

Vi sono poi bronzi di misura ridotta: il busto di Atena (Rodríguez Oliva 1978b: 43, n. 3) e il leone da Torrox; un piccolo braccio dalla villa El Ruedo; la testa di efebo (Vaquerizo 1995: 82-83; Baena 2004: 323) dalla villa de Las Minas; la pantera da Casilla de la Lámpara. Tali pezzi in qualche modo decoravano le case sotto forma di figurine o appliques al mobilio domestico come l'Ermafrodito danzante (Loza 1994: 269-273) dalla villa de El Ruedo. Si nota che per lo più si tratta di statue di dimensioni ridotte (dai $154 \mathrm{~cm}$ dell'efebo de la Finca Las Piletas ai $43 \mathrm{~cm}$ dell'Ermafrodito danzante da El Ruedo), raffiguranti divinità.

Seguono, secondo l'ordine quantitativo decrescente, i gruppi scultorei: tra questi di particolare pregio è da segnalare la rappresentazione di Perseo e Andromeda da El Ruedo (Vaquerizo e Noguera 1997: 118, n. 4; Beltrán e Loza 2003: 76, n. 9). L'iconografia di tale gruppo fa capo ad una composizione pittorica tardo-classica di cui ci dà notizia Plinio (Nat. Hist., XXXV, 130-133), attribuibile al pittore Nicia (330 a.C.), a sua volta a capo di una nutrita schiera di pitture pompeiane - ad esempio quella nella Casa dei Dioscuri (Helbig 1868: 186, $\mathrm{n}^{\circ} 1$ ). Il gruppo con Perseo e Andromeda documenta per la prima volta in ambito betico il motivo del pittore attico ed è la seconda scultura conosciuta basata sul mito del pittore Nicia.

Gli esempi pompeiani permettono di ricostruire l'iconografia dell'esemplare almedinillense: l'affresco pompeiano nella Casa dei Cinque Scheletri, oggi all'Antiquarium Comunale di Roma, mostra un uguale motivo e uno stile risalente al IV sec. d.C. (Mielsch 1978: 193-195, tav. 97, 1-2; AA.VV. 1981-1997, I, s.v. Andromeda: 781, $\mathrm{n}^{\circ} 72$ ).

In ambito teatrale rientrano gli oscilla decorati con maschere tragiche, comiche e satiresche, come possibile 
rimando all'uso di sospendere agli alberi, durante i riti dionisiaci, delle maschere del dio che dovevano creare nei peristili un'atmosfera da thiasos dionisiaco. L'antefissa con attore e maschera tragica dalla villa de la Estación (Romero et al. 2006: 251, fig. 8) e l'antefissa con maschera teatrale (Romero e Melero 2001: 240; Romero et al. 2006: 251) trovano dei paralleli - per il tipo di pettinato alto (onkos) che cade in blocchi di ciuffi simmetrici ad ambo i lati del volto - nei rilievi con maschere di Pompei; sono cronologicamente databili nel II sec. d.C. (Dwyer 1982: tav. XXVIII, n. 106 e tav. XIX, n. 109-110).

Il rilievo a disco (clipeo marmoreo o oscillum?) con ritratto maschile (Rodríguez Oliva 2004: 53, fig. 9; Romero et al. 2006: 254) proveniente dalla villa de la Estación può mostrare il ritratto del proprietario della villa. Le caratteristiche iconografiche seguono una cronologia che va dal 235 d.C. al 285 d.C. Il luogo di ritrovamento, accanto all'ingresso delle terme, fa pensare che fosse appeso alla porta. Si tratta, fino ad ora, del primo ritrovamento di una imago clipeata in situ nella penisola iberica.

Per quanto riguarda la ritrattistica ufficiale è da menzionare il busto cosiddetto di Druso (Baena 1984: 231-238) dalla villa della Estación, confrontabile con la testa bronzea di Druso trovata nel Tevere e conservata al Museo Nazionale Romano (Paribeni 1934: tav. 135). L'orientamento dritto delle ciocche della frangia, riscontrabile nell'esemplare del Museo Nazionale Romano e nel busto proveniente dalla Casa di Dedalo e Icaro di Pompei (AA.VV. 2008: 172, n. D15), si colloca bene nell'ambito della terza tipologia definita dallo Jucker (1977: 235, fig. 20b), corrispondente al secondo tipo (Tipo Béziers) della classificazione di Boschung (1993: 62-63, fig. $42 \mathrm{~Pb}$ ).

Sulla base di considerazioni di tipo iconografico è possibile stabilire la prevalenza tipologica di determinati soggetti nell'arredo marmoreo dei complessi abitativi (Cabra; Chirivel; Antequera; Montilla: Rodríguez Oliva 1993: 42; Vaquerizo 1995: 89-90; Aguilar de la Frontera: Koppel 1995: 34; Vaquerizo 1995: 82-83; Serrato: AA.VV. 1990: 100 e 253, n. 172; Manilva etc.).

Tali soggetti, per lo più dionisiaci sono chiaramente in relazione con la natura e concepiti per sfruttare e godere dell'otium e della vita privata, evocando un'ambientazione da paradeisos - ricreato spesso con la presenza importante dell'acqua e di fonti d'acqua pieno di piccoli animali e propizio al riposo - imparentato, in espressione senza dubbio tarda, ai fondamenti più puri della vecchia cultura ellenistica. Uno per tutti, l'eros dormiente proveniente dalla Casa di Mitra fa parte dei giochi d'acqua nel ninfeo centrale, secondo la ricostruzione di Loza (1993a: 101).

Piccole sculture riproducenti Fauni e Satirelli, esseri mitici, strettamente connessi con il mondo della natura e derivanti dalla scultura tardo ellenistica di origine alessandrina, sono elementi ornamentali molto diffusi anche nei giardini e nei peristili delle case pompeiane (AA.VV. 1996: 241; AA.VV. 2008: 84, n. B 18).

Sculture, rilievi, pitture e oggetti relazionati con il mondo dionisiaco rispondono ad una determinata estetica, in voga durante gli anni dell'Impero a Roma e nelle provincie, proveniente da una cultura mitologica e letteraria, di un'idea di sfruttamento e piacere. Così come è ammissibile, in alcuni casi, servirsi della figura di Dioniso come simbolo del suo otium, in altri lo è dei suoi negotia. Tale aspetto non evita che in determinate villae la disposizione dei gruppi particolarmente legati ad una divinità concreta, come Dioniso, possa essere relazionabile anche con la produzione agricola e zootecnica specifica del fundus, soprattutto se questo ha come base principale la viticoltura e la commercializzazione del prodotto vinificato.

Di riflesso, a Pompei, Dioniso era pienamente integrato in una città che faceva della produzione e del commercio del vino uno dei capisaldi della propria economia.

Le differenti tematiche analizzate, che coesistono negli ambienti delle villae hanno come obiettivo onorare il patronus, il pater familias, la domina o $\mathrm{i}$ benefattori della casa. Rientrano in questo gruppo le hermae ridotte a busti, offerte generalmente dai liberti e i ritratti realizzati ex professo da qualcuno dei membri della famiglia.

Vanno incluse tra le sculture che hanno un'intenzione colta e intellettuale i cicli delle nove muse, come mezzo per evocare il mondo della filosofia, della letteratura e delle arti. Eccezionale è il gruppo proveniente dalla villa malagueña di Churriana (Baena 2004: 326), costituito dalla figura di Urania (fig. 4) (Rodríguez de Berlanga 1995: 97-98, tav. IX; Corrales 2007: 208, fig. IV) e dalle teste di altre due Muse (Rodríguez Oliva 1995: 97-98, n. XIX, tav. XXVI), rinvenute nel ninfeo.

Infine sono attestate alcune statue con scopo puramente ornamentale, destinate ad esaltare il buon gusto del proprietario della casa: esempi eccellenti in Baetica sono le teste di Dioniso - Serrato e Aguilar de la Frontera - e il mellephebos anticariense (Loza 1996: 88; AA.VV. 2011).

Satirica signa utilizzati hortoque et foro, sono le hermae bacchiche o quelle che desiderano rimembrare 


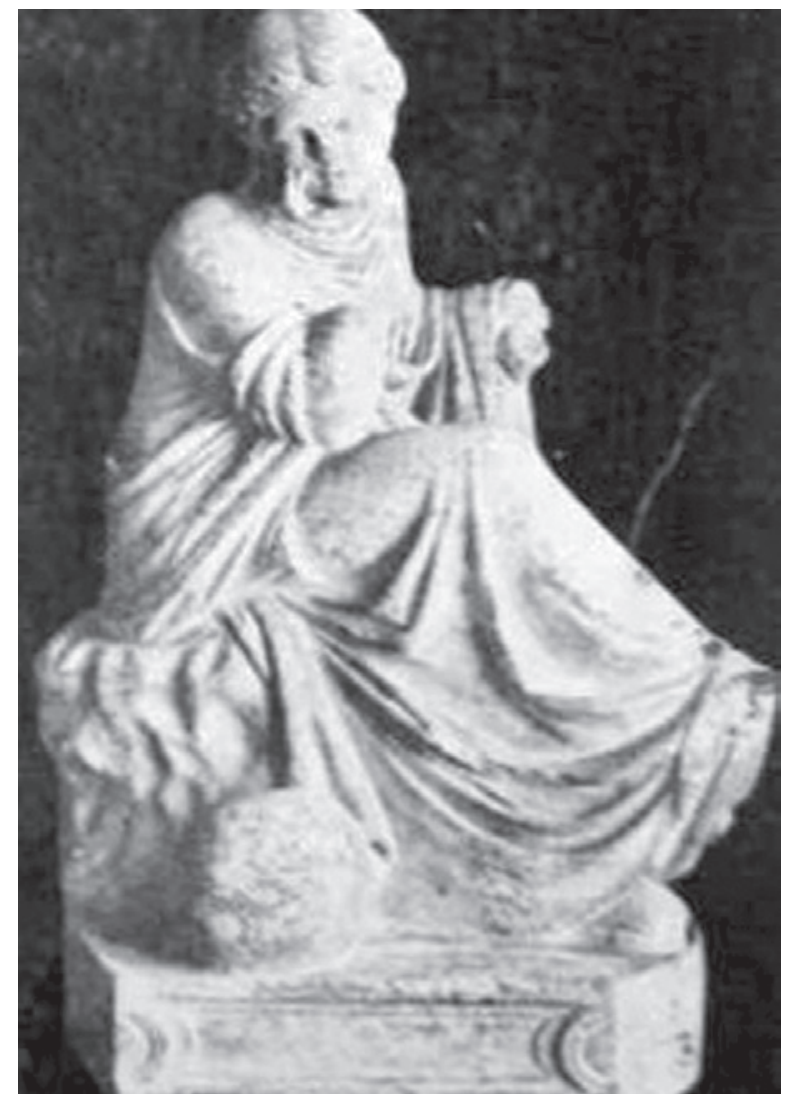

Figura 4. Figura di Urania

Alessandro Magno con un copricapo militare: le erme di Dioniso provenienti dalla villa de El Ruedo (Vaquerizo 1990: 133, n. 17 e 18; Vaquerizo e Noguera 1997: 136 ss., n. 7 e 8); le hermae bacchiche dalla villa di Benagalbón (Salado 2005: 353-378, fig. 20), e Sabinillas (Rodríguez Oliva 1979: 262-268, figg. 1-3); l'herma dionisiaca dalla villa de Villanueva de Algaidas (Corrales 2007: 207); l'herma da Alameda (Málaga), del tipo "testa di guerriero" (Baena 2002: 243-250).

Con scopo apotropaico le già citate sculture di Priapo portatore di frutti: Alameda e Finca las Piletas - Bobadilla. Per queste due sculture si propende per un rapporto - chiaramente ostentato dal fallo eretto - Priapo/ denaro, beneficio ottenuto mediante la protezione del dio a cui si deve il buon raccolto.

Nel tentativo di rendere più completa la trattazione riguardante l'arredo scultoreo delle villae baetiche sembra opportuno valutarne anche la collocazione effettiva all'interno dei singoli ambienti, evidenziando i rispettivi domini e significati e l'eventuale possibilità di riconoscere in questi cicli scultorei, apprestamenti meditati e/o programmi decorativi tout court.

Sulla base dei dati relativi ai luoghi di rinvenimento dei pezzi risulta che i principali ambienti che si decorano, insieme alle terme, sono i peristili, i giardini e in questi ultimi ninfei e viridaria.

Nel peristilio, il luogo più importante che introduce allo spazio privato vero e proprio, si manifesta in pieno il gusto decorativo; in esso la natura reale, rappresentata da aiuole, alberi e piante, si fonde con la decorazione scultorea più variegata. Popolano i giardini erme di Satiri, Sileni, Menadi, Pan, Dioniso, una serie di oscilla, elementi decorativi di forme e caratteristiche diverse, destinati ad essere sospesi tra gli intercolumni o ad essere sostenuti da colonnette decorative. In epoca tardoromana si uniscono a questi dei nuovi spazi quali i triclinia, i cubicula e la stanza di accoglienza al cliens.

Nella maggior parte dei casi la decorazione scultorea si unisce alle strutture acquatiche, per questo insieme alla tematica strettamente associata all'acqua - ninfe, statue/fiumi e altre personificazioni acquatiche - predomina il tema di Dioniso e il suo thiasos, che insieme ad altri personaggi come Priapo o fanciulli costituiscono, in molti casi, loro stessi delle statuefontana da cui zampilla l'acqua (Loza 1993a: 97-110; 1993b: 141-158). Tali ambienti domestici, strutturati intorno alle fontane o ai ninfei dei giardini e peristili, in cui si collocavano statue di ninfe o satiri, hanno precedenti nei gruppi decorativi di età ellenistica.

Per quanto riguarda la possibilità di riconoscere tra i gruppi scultorei delle villae raccolte la presenza di programmi decorativi, si riportano le parole della Koppel (1995: 48), secondo cui non si possono svincolare da un certo grado di standardizzazione molti di questi gruppi, aspetto che, unito alla sempre limitata offerta delle botteghe e, in definitiva, alla necessità di utilizzare gruppi e sculture dei secoli precedenti, fa concludere che "la decorazione scultorea riflette principalmente il gusto dell'epoca piuttosto che la personalità del proprietario". Un carattere collezionistico ed antiquario che evoca un'anelata cultura classica, motivo per cui è preferibile parlare di "collezioni" raccolte durante più generazioni o acquistate ex professo da appassionati di antiquariato, anziché programmi.

L'effetto di richiamo dell'ambiente privato al lusso e alla cultura - almeno supposta - dovette costituire uno degli scopi principali di questi domini del secolo IV d.C. - e forse anche prima - al momento di decorare le loro residenze di campagna. Un esempio è Cabra (Córdoba); in questa villa la presenza del gruppo che rappresenta il sacrificio del toro da parte di Mitra ha 
portato inizialmente ad accettare la presenza di un possibile mithraeum. Questa opinione è limitata dalla dinamica archeologica del sito, il cui scavo non ha restituito nessun materiale che possa far pensare ad una identificazione come tempio o luogo religioso. I suoi scavatori sono piuttosto convinti che le sculture, che rimontano al secolo II d.C., furono utilizzate con una finalità principalmente decorativa, come accadde per quelle della villa de El Ruedo. luogo.

Resta il problema di chi realizzò le opere e in quale

Seguendo un filone di ricerca attuale (Beltrán 1995: 216) - riguardante cave marmoree e botteghe di artigiani collegate agli insediamenti della Baetica - si menziona l'ipotesi secondo cui si tratti di pezzi creati in loco e distribuiti in zone più o meno limitrofe ai centri di estrazione dei marmi.

Nel caso della statuaria privata sembra possibile l'esistenza di artigiani itineranti o, in loro assenza, la domanda da parte dei committenti a botteghe fisse. Si ha notizia soprattutto per la città di Italica, tramite l'epigrafia, per tutto il II sec. a.C., della presenza di artigiani provenienti dal Mediterraneo orientale.

Quello che si è tentato di dimostrare in numerosi studi (Loza 1984-85: 131-136; Loza e Beltrán 1990; Vaquerizo 1995: 81-102; Beltrán e Loza 2001: $517-$ 546; 2003) è l'impiego della cava di marmo appartenente all'area di domanda di sculture (Mijas; Almadén de la Plata; Macael), nonostante sia evidente l'utilizzo di pietre come il giallo antico, quale il Pentelico (in cui sono alcuni pezzi di Almedinilla, perciò chiaramente importati) e quello di Paros, per l'esecuzione di molte delle opere ritrovate nel territorio betico. A queste vanno aggiunti i pezzi semielaborati importati dall'Italia.

L'uso del marmo potrebbe essersi sviluppato in luoghi con un insediamento consolidato, in cui tale materiale era riservato a determinati elementi architettonici come fusti, basi o cornici, decorazione parietale, opus sectile e soprattutto, l'ornamentazione scultorea. Tutto questo naturalmente delinea ambienti di lusso, in cui il marmo rappresenta un elemento molto apprezzato, di apparato.

Beltrán e Loza (2001: 536), occupandosi dei marmi bianchi malagueñi (cava di Mijas, del cui marmo fu fatta la maggior parte dei pezzi di Almedinilla) riferibili all'epoca alto imperiale, ritengono che le botteghe locali potevano situarsi in città dei dintorni, quali Suel, Cartima o Malaca, dal momento che soprattutto nelle ultime due sono documentati importanti programmi monumentali. L'origine locale del marmo sarebbe dunque la riprova della presenza di botteghe locali, situate probabilmente ai piedi della cava 0 , come già detto, nelle città vicine.

Il marmo locale sarà preferito ad esempio per una statua di Dioniso da Cabra, cronologicamente databile verso la metà circa del II sec. d.C. (Vaquerizo 1995: 85 , n. II.2) utilizzata nell'ultima fase della villa come decorazione da giardino con valore puramente decorativo e confrontabile con i pezzi conservati al Vaticano, Napoli e al Museo Torlonia (Lippold 1956: 254, n. 29, tav. 118).

Ancora, verrà utilizzato marmo locale per la divinità fluviale trovata in Santa Fe de los Boliches (Fuengirola), di cui è sconosciuta data e circostanza del ritrovamento. Si suppone che sia stata ritrovata nei pressi dell'Arroyo Real, un ambito prossimo alla villa de El Secretario: è forse ipotizzabile che il pezzo appartenga all'apparato scultoreo della stessa villa (Rodríguez Oliva 1978a: 382-385, lam. III,2; Beltrán e Loza 2001: 540 ss., n. 7).

Per i materiali apparsi in luoghi distanti c'è la possibilità che i pezzi si commercializzassero già elaborati oppure in blocchi successivamente lavorati nei luoghi di ricezione - ad esempio il caso di Almedinilla.

In conclusione la lettura dell'arredo scultoreo fa emergere l'immagine di una società che utilizza in ambito privato tali arredi come espressione di cultura, ricerca di natura, lusso e autorappresentazione del proprietario del fundus. La tematica resta tuttavia generale e convenzionale ed i motivi si ripetono spesso.

Risultano significativi l'impiego e le modalità corrispondenti all'idioma classico, la riproduzione del mondo greco attraverso copie e varianti, trasformazione dei opera nobilia, come manifestazione di una certa consapevolezza di valore culturale e intellettuale.

I pezzi ispirati a soggetti più antichi, quali le hermae (Campillos, Cortijo del Tajo, Benagalbón, Bobadilla), i satiri (Alameda, Bobadilla), o il gruppo di Perseo e Andromeda delle villa di Almedinilla, si attennero probabilmente all'offerta delle botteghe locali o officine specializzate (Zanker 1993: 188), che si conformava al grande sviluppo scultoreo dei secoli iniziali dell'impero.

Il continuo tentativo di imitazione riceve ulteriore conferma in un frammento di rilievo con pastore e coppia di buoi (Vaquerizo 1990: 132, n. 13; Vaquerizo e Noguera 1997: 196, n. 27) proveniente dalla villa de El Ruedo collocato verosimilmente in un ambiente di rappresentanza, riflesso dei pinakes delle grandi villae della capitale, dove venivano allestite piccole collezioni d'arte importate direttamente dalla Grecia. 
Ciò che risalta in questa sintesi sulla scultura romana proveniente da villae del territorio betico è che più volte si registra la presenza di copie di tipi rari, in taluni casi, esempi unici in tutto il territorio hispanico. È pertanto ipotizzabile che tali pezzi provengano direttamente da Roma, in un territorio legatissimo ai primi imperatori del II sec. d.C.

Da Italica proviene l'unica copia del cosiddetto atleta Amelung presente nelle provincie; di tale opera sono attualmente note le copie provenienti da Villa Adriana e da Porto (Slavazzi 2011: 561, fig. 3).

Le decorazioni parietali e pavimentali, gli arredi mobili e gli elementi quotidiani, quali mobilio e vasellame, contribuiscono a rinforzare lo status dei proprietari e la loro equiparazione alla élite imperiale; tali elementi vanno oltre i semplici canoni estetici per formare parte di quelli culturali, dell'essenza del modus vivendi romano che durante la seconda metà del I sec. d.C. e quello seguente impregnò le terre ispaniche, integrandole definitivamente nella civilizzazione romana.

L'intensificarsi della diffusione di villae nel periodo imperiale coincide, dal punto di vista cronologico, con la datazione attribuibile alla maggior parte degli apparati decorativi rinvenuti: l'arco cronologico dei pezzi scultorei inizia nel I sec. d.C., culmina nel II sec. d.C. (è il caso delle villae in provincia di Córdoba: Cercadilla; in provincia di Málaga: Bobadilla, Las Piletas, Alameda, Lacipo, Benagalbón) e si protrae sino al IV sec. d.C. Tale cronologia suggerisce l'idea di una produzione che avalla un uso consapevole in tutta la regione betica a partire da questa data (Beltrán 2004: 17-33), coincidente a sua volta, in territori come l'Alto Guadalquivir e la Campiña de Jaén, con il cospicuo processo di occupazione del territorio di epoca flavia.

\section{Ringranziamento}

Ringrazio il Prof. J. Beltrán Fortes (Dipartimento di Preistoria e Archeologia, Università di Siviglia) per il supporto accademico e morale fornitomi nello sviluppo di un soggiorno Erasmus presso l'Università di Siviglia nel 2008 durante la stesura della tesi di laurea specialistica (Università di Urbino), da cui l'articolo è tratto.

\section{BIBLIOGRAFIA}

AA.VV. (1981-1997): Lexicon Iconographicum Mythologiae Classicae, I-VIII. Zürich-München, Artemis \& Winkler Verlag.
AA.VV. (1990): Los bronces romanos en España. Catálogo de la Exposición. Madrid, Ministerio de Cultura.

AA.VV. (1996): Guzzo, P. G. (a cura di), Pompei. Abitare sotto il Vesuvio, Catalogo della mostra. Ferrara, Ferrara Arte.

AA.VV. (2008): Carrella, A.; D’Acunto, L. A.; Inserra, N. e Serpe, C. (a cura di), Marmora Pompeiana nel Museo Archeologico Nazionale di Napoli. Gli arredi scultorei delle case Pompeiane. Roma, Soprintendenza Archeologica di Pompei.

AA.VV. (2011): El Efebo de Antequera. Antequera, Proyectos 2007 Invehila.

Arce, J. (1986): "Dionysus-Bacchus in Roman Spain". Bulletin de Correspondance Hellénique 14: 168-174.

Atencia Páez, R. e Sola Márquez, A. (1978): “Arqueología romana malagueña: Fuengirola”. Jábega 23: 73-84.

Baena del Alcázar, L. (1980-81): “Dos esculturas de Príapo, inéditas, de la Vega de Antequera". Cuadernos de Prehistoria y Arqueología de la Universidad Autónoma de Madrid 7-8: 141-150.

Baena del Alcázar, L. (1984): "Un retrato julio-claudio en el Museo de Antequera", Actas II Congreso Andaluz de Estudios Clásicos: vol. II, 231-238. Málaga, Sociedad Española de Estudios Clásicos.

Baena del Alcázar, L. (1994): "La decoración escultórica en las ciudades romanas de la Baetica", en La ciudad en el mundo romano: Actas. XIV Congreso Internacional de Arqueología Clásica: vol. 2, 42-43. Tarragona (1993), Tarragona, Institut d'Estudis Catalans.

Baena del Alcázar, L. (2002): "Un nuevo hermes del tipo "cabeza de guerrero" de Alameda (Málaga)". Baetica 24: 243-250.

Baena del Alcázar, L. (2004): "La escultura culta en Hispania. Planteamientos teóricos", en T. Nogales y L. J. Gonçalves (eds.), Actas de la IV Reunión sobre Escultura Romana en Hispania: 321-338. Lisboa (2002), Madrid, Ministerio de Cultura.

Baena del Alcázar, L. (2007): "Hipótesis reconstructivas de esculturas romanas ideales de la Bética". Anales de Arqueología Cordobesa 18: 237-262.

Balil Illana, A. (1959-1960): “Centuriatio, observaciones sobre la parcelación y agrimensura romanas y su reconocimiento". Estudios Clásicos V: 346-359.

Beltrán Fortes, J. (1995): "La incorporación de los modelos griegos por las élites romanas en ámbito privado. Una aproximación arqueológica”, en E. Falque e F. Gascó (eds.), Graecia Capta. De la conquista de Grecia a la helenización de Roma: 201232. Huelva, Universidad de Huelva. 
Beltrán Fortes, J. (2004): “Opera nobilia en la escultura romana de la Bética”, en T. Nogales y L. J. Gonçalves (eds.), Actas de la IV Reunión sobre Escultura Romana en Hispania: 17-33. Lisboa (2002), Madrid, Ministerio de Cultura.

Beltrán Fortes, J. e Loza Azuaga, M.L. (2001): “El comercio de los mármoles blancos malagueños durante el alto imperio romano", en Comercio y comerciantes en la Historia Antigua de Málaga: 517-546. Málaga, CEDMA.

Beltrán Fortes, J. e Loza Azuaga, M.L. (2003): El mármol de Mijas. Explotación, comercio y uso en época antigua. Málaga, Museo Histórico Etnológico de Mijas.

Bieber, M. (1961): The Sculpture of the Hellenistic Age. New York, Columbia University Press.

Blanco, A.; García, J. e Bendala, M. (1972): "Excavaciones en Cabra (Córdoba). La Casa del Mitra (Primera campaña, 1972). Habis III: 297-319.

Boschung, D. (1993): Die Bildnisse des Augustus. Berlin, Gebruder Mann Verlag.

Budde, L. e Nicholls, R. (1964): A Catalogue of the Greek and Roman Sculpture in the Fitzwilliam Museum Cambridge. Cambridge, Cambridge University.

Carrillo Díaz-Pinés, J. R. (1993): "Los estudios sobre las villas romanas de Andalucía: una revisión historiográfica". Anales de Arqueología Cordobesa 4: 233-257.

Cerrillo Martín de Cáceres, E. (1995): "Reflexiones sobre las villae romanas en Hispania", en J. M. Noguera (ed.), Poblamiento rural romano en el Sureste de Hispania: 17-26. Jumilla (1993), Murcia, Universidad de Murcia.

Corrales Aguilar, P. (2007): "Las casas romanas malacitanas: simbolismo arquitectonico y decorativo". Anales de Arqueología Cordobesa 18: 201-218.

Corzo, R. e Jiménez, A. (1980): “Organización territorial de la Baetica". Archivo Español de Arqueología 53: 21-47.

De Nuccio, M. e L. Ungaro, L. (2002): I marmi colorati della Roma imperiale, Venezia, Electa.

Dwyer, E. J. (1982): Pompeian Domestic Sculpture. A study of five Pompeian Houses and their contents. Roma, Giorgio Bretschneider.

Fernández-Castro, M. C. (1982): Villas Romanas en España. Madrid, Ministerio de Cultura.

Fornell Muñoz, A. (2005): Las Villae Romanas en la Andalucía Mediterránea y del Estrecho. Jaén, Universidad de Jaén.

García y Bellido, A. (1952): “El Mithras tauróktonos de Cabra (Córdoba)". Archivo Español de Arqueología 25: 389-392.
Giuliani, F. C. (1966): Tibur, II (Forma Italiae I, 3). Roma, De Luca Editore.

Gorges, J.G. (1979): Les Villas Hispano-romaines. París, De Boccard.

Helbig, W. (1868): Wandgemälde der vom Vesuvverschütteten Städte Campaniens. Leipzig, Breitkopf \& Härte.

Jashemski, W. (1993): The Gardens of Pompeii. New Rochelle, Caratzas.

Jucker, H. (1977): "Die Prinzen des Statuenzyclus aus Velleia. Umfang und Datierung der Stiftung des L. Calpurnius Piso". Jahrbuch des Deutschen Archäologischen Instituts 92: 204-240.

Kaschnitz-von Weinberg, G. (1937): Sculture del Magazzino del Museo Vaticano. Città del Vaticano, G. Bardi.

Koppel, E.M. (1995): “La decoración escultórica de las villae romanas de Hispania", en J. M. Noguera (ed.), Poblamiento rural romano en el Sureste de Hispania: 27-48. Junilla (1993), Murcia, Universidad de Murcia.

Koppel, E.M. (1956): Die Skulpturen des Vatikanischen Museums, III (2). Berlin, Kommission bei G. Reimer.

Loza Azuaga, M.L. (1984-85): "Notas sobre la explotación del mármol blanco de la sierra de Mijas en época romana". Mainake VI-VII: 131-136.

Loza Azuaga, M.L (1993a): "La escultura de fuentes en Hispania: ejemplos de la Baetica", en T. Nogales (ed.), Actas de la I Reunión sobre Escultura Romana en Hispania: 97-110. Mérida (1992), Madrid, Ministerio de Cultura.

Loza Azuaga, M.L (1993b): "Estatuas-fuentes romanas de Colonia Patricia Corduba". Anales de Arqueología Cordobesa 4: 141-158.

Loza Azuaga, M.L (1994): "El Hermafrodita en bronce de la villa de Almedinilla (Córdoba)". Archivo Español de Arqueología 67: 269-273.

Loza Azuaga, M.L (1996): "Esculturas romanas en bronce del sur de la provincia de Córdoba", en J. Massó y P. Sada (eds.), Actas de la II Reunión sobre Escultura Romana en Hispania: 79-94. Tarragona (1994), Tarragona, Museo Nacional de Arqueología de Tarragona.

Loza Azuaga, M. L. e Beltrán Fortes, J. (1990): La explotación del mármol blanco de la sierra de Mijas en época romana. Bellaterra, Universidad Autónoma de Barcelona.

Mendoza Eguaras, A.; Salvatierra Cuenca, V.; Jabaloy Sánchez, M. E.; García Granados, J. A. e Toro Moyano, I. (1985): "Las termas de Lecrín (Granada). 
Avance de la $1^{\text {a }}$ campaña", en XVII Congreso Nacional de Arqueología. Actas: 897-902. Zaragoza, Ministerio de Cultura.

Michon, E. (1933): "Éphèbe couronné. Nouvelle statue de bronze trouvée à Volubilis". Monuments Piot 33: 119-134.

Mielsch, H. (1978): "Zur stadtrömischen Malerei des IV Jahrhunderts n. Chr.’. Römische Mitteilungen 85: 193-195.

Neudecker, R. (1988): Die Skulpturenausstattung römischer villen in Italien. Mainz am Rhein, P. von Zabern.

Paribeni, R. (1934): Il ritratto nell'arte antica. Milano, Treves.

Picard, C. (1939): La sculpture grecque, II(1). Paris, Picard.

Pochmarski, E. (1974): Das Bild des Dionysos in der Rundplastik der klassischen Zeit Griechland. Vienna, Verband der Wiss. Ges. Österreichs.

Ponsich, M. (1987): Implantation rurale antique sur le Bas-Guadalquivir III. Bujalance, Montoro, Andújar, Madrid, Boccard.

Portillo, R.; Rodríguez Oliva, P. e Stylow, A. U. (1985): "Porträthermen mit Inschrift im römischen Hispanien”. Madrider Mitteilungen 26: 184-217.

Posac Mon, C. e Rodríguez Oliva, P. (1979): "La villa romana de Sabinillas (Manilva)", Mainake I: 129145.

Rodríguez de Berlanga, M. (1995): Catalogo del Museo Loringiano. Málaga, Universidad de Málaga (reed.).

Rodríguez Oliva, P. (1977): "Las ruinas romanas del Faro de Torrox y el problema de Caviclum". Jábega 20: 11-26.

Rodríguez Oliva, P. (1978a): "Esculturas del conventus de Gades". Boletín del Seminario de Arte y Arqueología XLIV: 374- 385.

Rodríguez Oliva, P. (1978b): La villa romana del Faro de Torrox (Málaga). Valladolid, Universidad de Valladolid.

Rodríguez Oliva, P. (1979): "Esculturas del conventus de Gades (II)". Boletín del Seminario de Arte y Arqueología XLV: 258-269.

Rodríguez Oliva, P. (1987): Mosaicos romanos de Bobadilla (Málaga). Málaga, Diputación Provincial de Málaga.

Rodríguez Oliva, P. (1993): “Ciclos escultóricos en la casa y en la ciudad de la Bética”, en T. Nogales (ed.), Actas de la I Reunión sobre Escultura Romana en Hispania: 23-61. Mérida (1992), Madrid, Ministerio de Cultura.

Rodríguez Oliva, P. (2004): “Miscelánea de esculturas de la Bética”, en T. Nogales y L. J. Gonçalves (eds.), Actas de la IV Reunión sobre Escultura Romana en Hispania: 52-80. Lisboa (2002). Madrid, Ministerio de Cultura.

Rodríguez Oliva, P. (2010): "Nuevos hallazgos escultóricos en villae de los alrededores de Malaca y noticias sobre otras esculturas antiguas", en J.M. Abascal y R. Cebrián (eds.), Actas de la VI Reunión de Escultura Romana en Hispania: 61-96. Cuenca (2008). Murcia, Tabularium.

Romero Pérez, M. e Melero García, F. (2001): "La villa romana de la Estación. Antequera, Málaga". Revista de Estudios Antequeranos 12: 235.

Romero, M.; Mañas, I. e Vargas, S. (2006): "Primeros resultados de las excavaciones realizadas en la Villa de la Estación (Antequera, Málaga)". Archivo Espanol de Arqueología 79: 239-258.

Salado Escaño, J. B. (2005): "La villa romana de la Torre de Benagalbón, Málaga. Primera descripción”. Mainake XXVII: 353-378.

Santero Santurino, J. M. (1975): "Una villa tardo-romana en Paulenca (Guadix)". Noticiario Arqueológico Hispánico 3: 227-268.

Slavazzi, F. (2011): "Circolazione di tipi scultorei fra Roma e le provincie: su alcune copie di opere di età classica e sul loro «monopolio»", en Roma y las provincias: modelo y difusión: 559-565. Roma, L'Erma di Bretschneider.

Stuart Jones, H. (1968): A catalogue of the Ancient Sculptures preserved in the Municipal collections of Rome. The sculpture of the Palazzo dei Conservatori. Roma, L'Erma di Bretschneider (reed.).

Vaquerizo Gil, D. (1990): “La decoración escultórica de la villa romana de El Ruedo (Almedinilla, Córdoba)". Anales de Arqueología Cordobesa 1: 125-154.

Vaquerizo Gil, D. (1995): “El uso del mármol en la decoración arquitectónica y escultórica de villae cordobesas", J. M. Noguera (ed.), Poblamiento rural romano en el sureste de Hispania: 81-106. Jumilla (1993), Murcia, Universidad de Murcia.

Vaquerizo Gil, D. e Carrillo Díaz-Pines, J.R. (1995): "The Roman villa of El Ruedo (Almedinilla, Córdoba)". Journal of Roman Studies 8: 121-156.

Vaquerizo Gil, D. e Noguera Celdrán, J.M. (1997): La villa romana de El Ruedo (Almedinilla, Córdoba): decoración escultórica y interpretación. Córdoba, Universidad de Murcia.

Vorster Hesse, C. (1983): Griechische Kinderstatuen. Köln, Wasmuth.

Zanker, P. (1993): Pompei. Società, immagini urbane e forme dell'abitare. Torino, Einaudi. 